\title{
Similar Submodules and Coincidence Site Modules
}

\author{
P. ZEINER* \\ Fakultät für Mathematik, Universität Bielefeld, 33615 Bielefeld, Germany
}

\begin{abstract}
We consider connections between similar sublattices and coincidence site lattices and, more generally, between similar submodules and coincidence site modules of general (free) $\mathbb{Z}$-modules in $\mathbb{R}^{d}$. In particular, we generalise results obtained by S. Glied and M. Baake on similarity and coincidence isometries of lattices and certain lattice-like modules called $\mathcal{S}$-modules. An important result is that the factor group $\mathrm{OS}(M) / \mathrm{OC}(M)$ is Abelian for arbitrary $\mathbb{Z}$-modules $M$, where $\operatorname{OS}(M)$ and $\mathrm{OC}(M)$ are the groups of similar and coincidence isometries, respectively. In addition, we derive various relations between the indices of coincidence site lattices and their corresponding similar sublattices.
\end{abstract}

DOI: 10.12693/APhysPolA.126.641

PACS: $61.44 . \mathrm{Br}$

\section{Introduction}

Coincidence site lattices (CSLs) are an important tool in describing grain boundaries in crystals; see [1-5] and references therein. These concepts have been generalised for modules to analyse grain boundaries in quasicrystals $[6-9]$. On the other hand, similar sublattices and submodules have been studied [10-12], and it soon turned out that there must be close connections between these two types of sublattices, compare for instance [12] and [13] for similar sublattices and CSLs of the $A_{4}$-lattice. In 2008, Glied and Baake established a connection between similar sublattices and CSLs by showing that the group of coincidence isometries is a normal subgroup of the group of similarity isometries [14], a result which was later generalised to a certain class of modules [15], which the author called $\mathcal{S}$-modules.

In this paper, we want to have a closer look at these connections. In the first part, we elaborate in more detail on the connections between similar sublattices and CSLs by proving some relation between the coincidence index and the so-called denominator of a coincidence isometry. In the second part, we present a generalisation of the results by Glied and Baake to general $\mathbb{Z}$-modules.

Let us fix some notations and recall the most important notions first, for more details we refer to $[7,14]$. Throughout this paper, $\Lambda \subset \mathbb{R}^{d}$ denotes a lattice of full rank in $\mathbb{R}^{d}$. An isometry $R \in \mathrm{O}(d, \mathbb{R})$, i.e. an orthogonal transformation in $\mathbb{R}^{d}$, is called a coincidence isometry of $\Lambda$ if the intersection $\Lambda \cap R \Lambda$ is a sublattice of $\Lambda$ of full rank. This happens if and only if the index $\Sigma_{\Lambda}(R):=[\Lambda: \Lambda \cap R \Lambda]$, the so-called coincidence index, is finite. In this case, we call $\Lambda(R):=\Lambda \cap R \Lambda$ a coincidence site lattice (CSL). The set of all coincidence isometries forms a group, which we denote by $\mathrm{OC}(\Lambda)$.

Two lattices $\Lambda_{1}$ and $\Lambda_{2}$ are called commensurate, denoted by $\Lambda_{1} \sim \Lambda_{2}$, if $\Lambda_{1} \cap \Lambda_{2}$ is a sublattice of full rank of both $\Lambda_{1}$ and $\Lambda_{2}$. As we assume throughout this paper

*e-mail: pzeiner@math.uni-bielefeld.de that any lattice has full rank, $\Lambda_{1}$ and $\Lambda_{2}$ are commensurate if and only if $\Lambda_{1} \cap \Lambda_{2}$ is a sublattice of at least one of $\Lambda_{1}$ and $\Lambda_{2}$. Equivalently, $\Lambda_{1}$ and $\Lambda_{2}$ are commensurate if and only if there exists an integer $m$ such that $m \Lambda_{1} \subset \Lambda_{2}$. Thus, $R$ is a coincidence isometry of $\Lambda$ if and only if $\Lambda$ and $R \Lambda$ are commensurate.

A similar sublattice (SSL) is a sublattice of $\Lambda$ that is similar to $\Lambda$, i.e. it is a sublattice of the form $\alpha R \Lambda \subset \Lambda$ for some $R \in \mathrm{O}(d, \mathbb{R})$ and $\alpha \in \mathbb{R}^{+}$. We call $R$ a similarity isometry of $\Lambda$, if there exists an $\alpha \in \mathbb{R}^{+}$such that $\alpha R \Lambda$ is a similar sublattice. The set of similarity isometries forms a group as well, which we denote by $\operatorname{OS}(\Lambda)$. For any $R \in \operatorname{OS}(\Lambda)$ we define the denominator $\operatorname{den}_{\Lambda}(R)$ as the smallest scaling factor $\alpha \in \mathbb{R}^{+}$such that $\alpha R \Lambda \subset \Lambda$. Recall that $\alpha^{d}$, and thus $\operatorname{den}_{\Lambda}(R)^{d}$, is an integer.

For any $R \in \mathrm{O}(d, \mathbb{R})$ we can define the two sets:

$$
\begin{aligned}
& \operatorname{Scal}_{\Lambda}(R):=\{\alpha \in \mathbb{R} \mid \alpha R \Lambda \subseteq \Lambda\}, \\
& \operatorname{scal}_{\Lambda}(R):=\{\alpha \in \mathbb{R} \mid \alpha R \Lambda \sim \Lambda\},
\end{aligned}
$$

where the first one consists of all scaling factors giving rise to a similar sublattice and the latter is the set of all scaling factors which lead to lattices commensurate to $\Lambda$. Observe that $\operatorname{Scal}_{\Lambda}(R)$ and $\operatorname{scal}_{\Lambda}(R)$ are non-trivial if and only if $R \in \mathrm{OS}(\Lambda)$. In other words, $\operatorname{Scal}_{\Lambda}(R) \neq$ $\{0\}$, and likewise $\operatorname{scal}_{\Lambda}(R) \neq\{0\}$, if and only if $R \in$ $\operatorname{OS}(\Lambda)$, compare $[14,15]$. In particular, if $E$ is the identity operation, then $\operatorname{Scal}_{\Lambda}(E)=\mathbb{Z}$ and $\operatorname{scal}_{\Lambda}(E)=\mathbb{Q}$. More generally,

$$
\begin{aligned}
& \operatorname{Scal}_{\Lambda}(R)=\operatorname{den}_{\Lambda}(R) \mathbb{Z}, \\
& \operatorname{scal}_{\Lambda}(R)=\operatorname{den}_{\Lambda}(R) \mathbb{Q} .
\end{aligned}
$$

\section{Similar sublattices and CSLs}

As mentioned above, there is a close connection between the groups $\mathrm{OC}(\Lambda)$ and $\operatorname{OS}(\Lambda)$. In particular, Glied and Baake have shown the following [14] result.

$$
\begin{aligned}
& \text { Theorem 1. The kernel of the homomorphism } \\
& \qquad \begin{array}{l}
\phi: \operatorname{OS}(\Lambda) \rightarrow \mathbb{R}^{+} / \mathbb{Q}^{+} \\
R \mapsto \operatorname{scal}_{\Lambda}(R) \cap \mathbb{R}^{+}
\end{array}
\end{aligned}
$$

is the group $\mathrm{OC}(\Lambda)$. Thus $\mathrm{OC}(\Lambda)$ is a normal subgroup of $\operatorname{OS}(\Lambda)$ and $\operatorname{OS}(\Lambda) / \mathrm{OC}(\Lambda)$ is Abelian. Moreover, all el- 
ements of $\mathrm{OS}(\Lambda) / \mathrm{OC}(\Lambda)$ have finite order, in particular, their order is a divisor of the dimension d.

Hence, any coincidence isometry is a similarity isometry, and thus it makes sense to compare $\Sigma(R)$ and den $(R)$ for any $R \in \mathrm{OC}(\Lambda)$. By definition, $\Sigma(R)$ is a positive integer, and so is $\operatorname{den}(R)$ for any $R \in \mathrm{OC}(\Lambda)$. This can be seen as follows: by Theorem 1 , we see $\operatorname{scal}_{\Lambda}(R)=\mathbb{Q}$, which reflects the fact that $\Lambda$ and $R \Lambda$ are commensurate. Thus $\operatorname{den}(R) \in \operatorname{Scal}_{\Lambda}(R) \subset \operatorname{scal}_{\Lambda}(R)=\mathbb{Q}$, and as $\operatorname{den}(R)^{d} \in \mathbb{N}$, we see $\operatorname{den}(R) \in \mathbb{N}$.

Recall that $\Sigma\left(R^{-1}\right)=\Sigma(R)$ for any $R \in \mathrm{OC}$, compare [7], whereas $\operatorname{den}\left(R^{-1}\right)$ and $\operatorname{den}(R)$ are not equal in general $[14,18]$. Nevertheless $\operatorname{den}\left(R^{-1}\right)$ and $\operatorname{den}(R)$ are not independent of each other, as we will show in a moment. But first we mention

Lemma 1. Let $\Lambda$ be a lattice in $R^{d}$. Then, for any $R, S \in \mathrm{OS}(\Lambda)$,

$$
\frac{\operatorname{den}(R) \operatorname{den}(S)}{\operatorname{den}(R S)} \in \mathbb{N} .
$$

Proof. By the definition of the denominator,

$$
\begin{aligned}
& \operatorname{den}(R) \operatorname{den}(S) R S \Lambda=\operatorname{den}(R) R(\operatorname{den}(S) S \Lambda) \\
& \quad \subseteq \operatorname{den}(R) R \Lambda \subseteq \Lambda
\end{aligned}
$$

which is only possible if $\operatorname{den}(R) \operatorname{den}(S) \in \operatorname{Scal}(R S)=$ $\operatorname{den}(R S) \mathbb{Z}$. As the denominator is positive by definition, Eq. (5) follows.

Now we are able to prove the following relations between $\operatorname{den}\left(R^{-1}\right)$ and $\operatorname{den}(R)$.

Lemma 2. Let $\Lambda$ be a lattice in $\mathbb{R}^{d}$. Then, for any $R \in \mathrm{OS}(\Lambda)$,

$$
\begin{aligned}
& \operatorname{den}(R) \operatorname{den}\left(R^{-1}\right) \in \mathbb{N}, \\
& \frac{\operatorname{den}(R)^{d-1}}{\operatorname{den}\left(R^{-1}\right)} \in \mathbb{N} .
\end{aligned}
$$

Proof. Eqation (6) is just a special case of Lemma 1, with $S=R^{-1}$ and $\operatorname{den}(E)=1$.

For the second claim, observe that $\operatorname{den}(R) R \Lambda$ has index $[\Lambda: \operatorname{den}(R) R \Lambda]=\operatorname{den}(R)^{d}$ in $\Lambda$. Thus, $\operatorname{den}(R)^{d} \Lambda \subseteq$ $\operatorname{den}(R) R \Lambda$, or equivalently, $\operatorname{den}(R)^{d-1} R^{-1} \Lambda \subseteq \Lambda$. This means $\operatorname{den}(R)^{d-1} \in \operatorname{Scal}_{\Lambda}(R)$, and now Eq. (3) implies Eq. (6).

For $d=2$ this result simplifies considerably.

Corollary 1. $\operatorname{den}_{\Lambda}\left(R^{-1}\right)=\operatorname{den}_{\Lambda}(R)$ for any planar lattice $\Lambda$ and any $R \in \operatorname{OS}(\Lambda)$.

We are now able to prove the following bounds for $\Sigma(R)$ in terms of the denominators $\operatorname{den}(R)$ and $\operatorname{den}\left(R^{-1}\right)$.

Theorem 2. Let $\Lambda$ be a lattice in $\mathbb{R}^{d}$. Then, for any $R \in \mathrm{OC}(\Lambda)$,

1. $\operatorname{lcm}\left(\operatorname{den}_{\Lambda}(R), \operatorname{den}_{\Lambda}\left(R^{-1}\right)\right)$ divides $\Sigma_{\Lambda}(R)$,
2. $\Sigma_{\Lambda}(R)$ divides $\operatorname{gcd}\left(\operatorname{den}_{\Lambda}(R), \operatorname{den}_{\Lambda}\left(R^{-1}\right)\right)^{d}$,

3. $\Sigma_{\Lambda}(R)^{2}$ divides $\operatorname{lcm}\left(\operatorname{den}_{\Lambda}(R), \operatorname{den}_{\Lambda}\left(R^{-1}\right)\right)^{d}$.

Proof. For (1) recall that $\Lambda(R)$ has index $\Sigma(R)$ in $\Lambda$, thus $\Sigma(R) \Lambda \subseteq \Lambda(R) \subseteq R \Lambda$, or equivalently, $\Sigma(R) R^{-1} \Lambda \subseteq \Lambda$. An argument as above shows that $\Sigma(R)$ is a multiple of $\operatorname{den}\left(R^{-1}\right)$. By symmetry, $\operatorname{den}(R)$ is a divisor of $\Sigma\left(R^{-1}\right)=\Sigma(R)$ as well, and hence (1) follows.

For (2) we exploit that $\operatorname{den}(R)$ is an integer for $R \in$ $\mathrm{OC}(\Lambda)$. Thus, $\operatorname{den}(R) R \Lambda$ is a sublattice of both $\Lambda$ and $R \Lambda$, and hence, $\operatorname{den}(R) R \Lambda \subseteq \Lambda(R)$. Comparing the indices of $\operatorname{den}(R) R \Lambda$ and $\Lambda(R)$ in $\Lambda$ shows that $\Sigma(R)$ divides $\operatorname{den}(R)^{d}$. Using $\Sigma\left(R^{-1}\right)=\Sigma(R)$ as above finally yields (2). Finally, let $a:=\operatorname{lcm}\left(\operatorname{den}(R), \operatorname{den}\left(R^{-1}\right)\right)$. Then $a \Lambda$ and $a R \Lambda$ are both sublattices of $\Lambda$ and $R \Lambda$, hence $a(\Lambda+R \Lambda)$ is a sublattice of $\Lambda \cap R \Lambda$ with index

$$
[R \cap R \Lambda: a(\Lambda+R \Lambda)]=\frac{a^{d}}{\Sigma(R)^{2}},
$$

as $\Sigma(R)=[\Lambda: \Lambda(R)]=[\Lambda+R \Lambda: \Lambda]$. Hence $\Sigma(R)^{2}$ divides $a$.

The situation becomes particularly simple for planar lattices, where we get the following result by recalling $\operatorname{den}_{\Lambda}(R)=\operatorname{den}_{\Lambda}\left(R^{-1}\right)$.

Corollary 2. Let $\Lambda$ be a lattice in $\mathbb{R}^{2}$. Then, for any $R \in \mathrm{OC}(\Lambda)$,

$$
\Sigma_{\Lambda}(R)=\operatorname{den}_{\Lambda}(R) .
$$

This result turns out to be very useful in the analysis of CSLs of planar lattices, as the denominator is usually much simpler to determine than the coincidence index.

In more than two dimensions Eq. (8) is in general not true anymore [7, 13, 16], although it may be satisfied in special cases, e.g. Eq. (8) holds for all coincidence isometries of the cubic lattices $[5,7,17]$.

One word of caution should be added. Although there are a lot of connections between CSLs and similar sublattices, a CSL is in general not a similar sublattice, see [17] for the cubic case. An exception are the square and hexagonal lattices $[6,7]$, where every CSL is a similar sublattice. In these two cases any coincidence rotation $R$ is the square of a suitable $S \in \mathrm{OS}(\Lambda)$ and we have $\Lambda(R)=$ $\operatorname{den}(S) S \Lambda$. But note that we cannot have $\Lambda(R)=\alpha R \Lambda$ except for symmetry operations $R$, since index considerations immediately imply $\alpha=\operatorname{den}(R)=\operatorname{den}\left(R^{-1}\right)$, which would give $\Sigma(R)=\operatorname{lcm}\left(\operatorname{den}(R), \operatorname{den}\left(R^{-1}\right)\right)^{d}$, which contradicts part (3) of Theorem 2 .

\section{The module case}

For the description of quasicrystals we need to go beyond lattices. The right tool here are special kinds of $\mathbb{Z}$-modules, namely free $\mathbb{Z}$-modules in $\mathbb{R}^{d}$, which we assume to span $\mathbb{R}^{d}$. More precisely, let $t_{1}, \ldots, t_{k} \in \mathbb{R}^{d}$ be rationally independent vectors that $\operatorname{span} \mathbb{R}^{d}$, i.e., $\left\langle t_{1}, \ldots, t_{k}\right\rangle_{\mathbb{R}}=\mathbb{R}^{d}$. Then

$$
M:=\left\langle t_{1}, \ldots, t_{k}\right\rangle_{\mathbb{Z}}=\left\{n_{1} t_{1}+\ldots+n_{k} t_{k} \mid n_{k} \in \mathbb{Z}\right\} \subseteq \mathbb{R}^{d}
$$


is called a (free) $\mathbb{Z}$-module of rank $k$ in dimension $d$.

Throughout this paper we shall call these $\mathbb{Z}$-modules simply modules. Clearly, $M$ is a lattice if and only if $k=d$. Only in this case $M$ forms a discrete subset of $\mathbb{R}^{d}$.

$M$ is a free Abelian group of rank $k$, i.e., it is isomorphic to $\mathbb{Z}^{k}$. In fact, $M$ can always be obtained as a projection of a $k$-dimensional lattice into $\mathbb{R}^{d}$. From an algebraic point of view, lattices and free $\mathbb{Z}$-modules of finite rank are the same, and thus we expect that concepts and results for similar sublattices and CSLs can be generalised easily to the case of modules. However, some care is needed as this problem is not a purely algebraic problem but also involves geometry. In fact, a key ingredient are orthogonal and similarity transformations in $\mathbb{R}^{d}$, which induce linear transformations in $\mathbb{R}^{k}$, but the latter need not be orthogonal or similarity transformations, respectively. In addition, the fact that $M$ is not discrete (except for lattices) may cause some problems. So we have to carefully check which concepts and results we can generalise.

We start with some definitions that are straightforward generalisations of the lattice case. We again restrict to submodules that have full rank, i.e. we call a module $M_{1} \subseteq M$ a submodule of $M \subseteq \mathbb{R}^{d}$, if and only if it has full rank, or equivalently, if the index $\left[M: M_{1}\right]$ is finite.

Definition 1. Two modules $M_{1}, M_{2} \subseteq \mathbb{R}^{d}$ are called commensurate, denoted by $M_{1} \sim M_{2}$, if $M_{1} \cap M_{2}$ is a submodule of both $M_{1}$ and $M_{2}$.

In other words, two modules $M_{1}, M_{2} \subseteq \mathbb{R}^{d}$ are called commensurate, if there exists an $m \in \mathbb{N}$ such that $m M_{1} \subseteq M_{2}$ and $m M_{2} \subseteq M_{1}$.

Two modules $M_{1}, \bar{M}_{2} \subseteq \mathbb{R}^{d}$ are called similar, if there exists a similarity transformation between them, i.e., there exist $\alpha \in \mathbb{R}$ and $R \in \mathrm{O}(d, \mathbb{R})$ such that $M_{1}=\alpha R M_{2}$.

Definition 2. $M_{1}$ is called a similar submodule (SSM) of $M$, if there exist $\alpha \in \mathbb{R}$ and $R \in \mathrm{O}(d, \mathbb{R})$ such that $M_{1}=\alpha R M$.

As in the lattice case we define the set of similarity isometries by

$$
\mathrm{OS}(M):=\left\{R \in \mathrm{O}(d, \mathbb{R}) \mid \exists \alpha \in \mathbb{R}^{+} \text {with } \alpha R M \subseteq M\right\} .
$$

As is to be expected from the lattice case we have

Theorem 3. $\mathrm{OS}(M) \subseteq \mathrm{O}(d, \mathbb{R})$ is a group.

The next quantity to look at are the scaling factors. In particular, it is the sets of scaling factors that are crucial for the understanding of SSMs and their relation to CSMs. Hence we define

$$
\begin{aligned}
& \operatorname{Scal}_{M}(R):=\{\alpha \in \mathbb{R} \mid \alpha R M \subseteq M\}, \\
& \operatorname{scal}_{M}(R):=\{\alpha \in \mathbb{R} \mid \alpha R M \sim M\} .
\end{aligned}
$$

We have already encountered them in the lattice case, but there their importance may not have been so clear as they were just multiples of the sets $\mathbb{Z}$ and $\mathbb{Q}$. Again, $\operatorname{Scal}_{M}(R)$ and $\operatorname{scal}_{M}(R)$ are non-trivial if and only if $R \in \operatorname{OS}(M)$, i.e. $\operatorname{Scal}_{M}(R) \neq\{0\}$, and $\operatorname{scal}_{M}(R) \neq\{0\}$, if and only if $R \in \mathrm{OS}(M)$.

Naturally, there are some restrictions on the possible values of $\alpha$. We have seen that $\alpha^{d} \in \mathbb{Z}$ for any $\alpha \in$ $\operatorname{Scal}_{\Lambda}(R)$ in the case of lattices. More generally, one can show $\alpha^{d} \in \mathcal{S}$, if $M$ is an $\mathcal{S}$-module [15]. In general, the situation is more complex, and the crucial quantity is the rank $k$.

Theorem 4. Any $\alpha \in \operatorname{Scal}_{M}(R)$ is an algebraic integer. If $M$ has rank $k$, then $\alpha$ has degree at most $k(k-1)$.

For lattices and $\mathcal{S}$-modules the degree of $\alpha$ is bounded by $k$, whereas in general the upper bound $k(k-1)$ cannot be improved, as is shown by the following example.

Example 1. Let $\eta=\mathrm{e}^{\frac{\mathrm{i} \pi}{3}} \sqrt[3]{\tau}-\mathrm{e}^{-\frac{\mathrm{i} \pi}{3}} \frac{1}{\sqrt[3]{\tau}}$, where $\tau=$ $\frac{1+\sqrt{5}}{2}$ is the golden mean. Then $M=\mathbb{Z}[\eta]$ has rank 3 , as $\eta$ satisfies $\eta^{3}+3 \eta-1=0$. Here, $\eta=|\eta| \frac{\eta}{|\eta|}$ is a symmetry operation, whose scaling factor has degree $6=3 \cdot 2$.

Let us go a step further and ask which properties the sets $\operatorname{Scal}(R)$ and $\operatorname{scal}(R)$ have. To begin with, we consider $\operatorname{Scal}(E)$, which gives the "trivial" CSMs. As $\operatorname{Scal}(E)=\mathbb{Z}$ for lattices, and $\operatorname{Scal}(E)=\mathcal{S}$ for $\mathcal{S}$-modules, we expect $\operatorname{Scal}(E)$ to be a ring of algebraic integers.

Theorem 5. Let $M \subseteq \mathbb{R}^{d}$ be a free $\mathbb{Z}$-module of rank $k$. $\operatorname{Scal}_{M}(E)$ is a ring of some algebraic integers. In particular, $\operatorname{Scal}_{M}(E)$ is a ring with unity and it is a finitely generated free $\mathbb{Z}$-module, whose rank is a divisor of $k$ and is at most $\frac{k}{d}$. Moreover, $\operatorname{scal}(E)$ is the corresponding field of quotients.

Hence the modules $M$ are not only free $\mathbb{Z}$-modules, but also $\operatorname{Scal}(E)$-modules, but in general not free ones, as is shown by the following example.

Example 2. Let $\xi_{8}=\mathrm{e}^{\mathrm{i} \pi / 4}$. Then $M=$ $\left\langle 1, \mathrm{i}, 2 \xi_{8},-2 \bar{\xi}_{8}\right\rangle_{\mathbb{Z}}$ is a submodule of $\mathbb{Z}\left[\xi_{8}\right]$ of index 4 . In particular, $\operatorname{Scal}_{M}(E)=\mathbb{Z}[2 \sqrt{2}]$, which is not a principle ideal domain (PID). $M$ is not a free $\operatorname{Scal}_{M}(E)$-module and thus not an $\mathcal{S}$-module in the sense of [15].

We now look at $\operatorname{Scal}(R)$ and $\operatorname{scal}(R)$ for general $R$. We start with $\operatorname{scal}(R)$, as the results are much nicer for $\operatorname{scal}(R)$, which is due to the fact that $\operatorname{scal}(E)$ is a field.

Theorem 6. Let $R, S \in \mathrm{OS}(M)$ and let $\alpha$ be an arbitrary element of $\operatorname{scal}_{M}(R)$. Then,

$$
\begin{aligned}
& \operatorname{scal}_{M}(R)=\alpha \operatorname{scal}_{M}(E), \\
& \operatorname{scal}_{M}(R S)=\operatorname{scal}_{M}(R) \operatorname{scal}_{M}(S) .
\end{aligned}
$$

In fact, this is in some sense a generalisation of Lemma 1 and Eq. (4). In particular, it follows that:

$$
\operatorname{scal}_{M}(R) \operatorname{scal}_{M}\left(R^{-1}\right)=\operatorname{scal}_{M}(E) \text {. }
$$

Thus, the set $\{\operatorname{scal}(R) \mid R \in \mathrm{OS}(M)\}$ has a natural group structure, with unit element $\operatorname{scal}_{M}(E)$. It is isomorphic to a (countable) subgroup of a factor group of the multiplicative group $\left(\mathbb{R}^{+}, \cdot\right)$. It will turn out later that this 
group plays a fundamental role in connecting $\mathrm{OC}(M)$ and $\operatorname{OS}(M)$.

For $\operatorname{Scal}(R)$ the situation is more complex, and the generalisation of Eq. (3) reads as follows.

Theorem 7. $\operatorname{Scal}_{M}(R)$ is a finitely generated free $\mathbb{Z}$ module. Moreover, $\beta \operatorname{Scal}_{M}(R) \subseteq \operatorname{Scal}_{M}(R)$ for any $\beta \in \operatorname{Scal}_{M}(E)$ i.e., $\operatorname{Scal}_{M}(R)$ is also a finitely generated $\operatorname{Scal}_{M}(E)$-module.

If $\operatorname{Scal}_{M}(E)$ is a PID, $\operatorname{Scal}_{M}(R)$ is a free $\operatorname{Scal}_{M}(E)$ module of rank 1 , i.e. there exists an $\alpha \in \operatorname{Scal}_{M}(R)$ such that $\operatorname{Scal}_{M}(R)=\alpha \operatorname{Scal}_{M}(E)$.

Thus, defining a denominator makes only sense if $\operatorname{Scal}_{M}(E)$ is a PID. Nevertheless, one can generalise Lemma 1 and 2 to a certain extent using ideals [18]. However, we do not want to pursue this topic here any further, as some complications arise if $M$ is not a free $\operatorname{Scal}_{M}(E)$-module.

Let us turn our attention to coincidence site modules now, which are defined as follows.

Definition 3. Let $R \in \mathrm{O}(d, \mathbb{R})$. If $M$ and $R M$ are commensurate, $M(R):=M \cap R M$ is called a coincidence site module (CSM). In this case, $R$ is called a coincidence isometry. The corresponding index $\Sigma_{M}(R):=$ $[M: M(R)]$ is called its coincidence index.

In complete analogy to the lattice case we have the following result.

Theorem 8. The set of all coincidence isometries

$$
\mathrm{OC}(M):=\{R \in \mathrm{O}(d, \mathbb{R}) \mid M \sim R M\}
$$

forms a group, a subgroup of $\mathrm{O}(d, \mathbb{R})$.

As $M$ is not discrete in general, we cannot define a unit cell with a non-zero volume. Thus, the proof of the following result becomes more complicated, as one needs algebraic methods instead of the usual argument of the preservation of volume [18].

Theorem 9. For any $R \in \mathrm{OC}(M)$

$$
\Sigma_{M}(R)=\Sigma_{M}\left(R^{-1}\right) .
$$

As for lattices, the group $\mathrm{OC}(M)$ can be characterised by $\operatorname{scal}_{M}(R)$.

Lemma 3. Let $M \subseteq \mathbb{R}^{d}$ be a finitely generated $\mathbb{Z}$-module and let $\mathrm{O}(M)$ be its symmetry group. Then

1. $R \in \mathrm{OC}(M)$ if and only if $1 \in \mathrm{scal}_{M}(R)$.

2. $R \in \mathrm{O}(M)$ if and only if $1 \in \mathrm{Scal}_{M}(R)$.

By Theorem 6, $\{\operatorname{scal}(R): R \in \mathrm{OS}(M)\}$ forms a group with unit element $\operatorname{scal}(E)$. This gives us the following analogue of Theorem 1
Theorem 10. The kernel of the homomorphism

$$
\phi: \mathrm{OS}(M) \rightarrow \mathbb{R}^{+} /\left(\operatorname{scal}_{M}(E) \cap \mathbb{R}^{+}\right),
$$

$$
R \mapsto \operatorname{scal}_{M}(R) \cap \mathbb{R}^{+}
$$

is the group $\mathrm{OC}(M)$. Thus $\mathrm{OC}(M)$ is a normal subgroup of $\mathrm{OS}(M)$ and $\mathrm{OS}(M) / \mathrm{OC}(M)$ is Abelian.

We have seen that in the lattice case all elements of $\operatorname{OS}(M) / \mathrm{OC}(M)$ have finite order, which is a divisor of $d$. This is no longer true for general modules $M$, see Example 1 , where there are no non-trivial coincidence isometries and any element of $\mathrm{OS}(M) / \mathrm{OC}(M)$, except the unit element, has infinite order.

\section{Acknowledgments}

The author thanks M. Baake and S. Glied for interesting discussions. This work was supported by the German Research Council (DFG), within the CRC 701.

\section{References}

[1] G. Friedel, Leçons de Cristallographie, Blanchard, Paris 1911.

[2] M.L. Kronberg, F.H. Wilson, Trans. A.I.M.E. 185 501 (1949).

[3] S. Ranganathan, Acta Crystallogr. 21, 197 (1966).

[4] W. Bollmann, Crystal Defects and Crystalline Interfaces, Springer, Berlin 1970.

[5] H. Grimmer, W. Bollmann, D. H. Warrington, Acta Crystallogr. A 30, 197 (1974).

[6] P.A.B. Pleasants, M. Baake, J. Roth, J. Math. Phys. 37, 1029 (1996).

[7] M. Baake, in: The Mathematics of Long-Range Aperiodic Order, Ed.: R.V. Moody, Kluwer, Dordrecht 1997, p. 9.

[8] D.H. Warrington, Mater. Sci. Forum 126-128, 57 (1993).

[9] D.H. Warrington, R. Lück, in Proc. Intl. Conf. on Aperiodic Crystals (Les Diablerets), Eds.: G. Chapuis, W. Paciorek, World Scientific, Singapore 1994, p. 30 .

[10] J.H. Conway, E.M. Rains, N.J.A. Sloane, Can. J. Math. 51, 1300 (1999).

[11] M. Baake, U. Grimm, Z. Kristallogr. 219, 72 (2004).

[12] M. Baake, M. Heuer, R.V. Moody, J. Algebra 320 , 1391 (2008)

[13] M. Baake, M. Heuer, U. Grimm, P. Zeiner, Europ. J. Combinatorics 29, 1808 (2008).

[14] S. Glied, M. Baake, Z. Kristallogr. 223, 770 (2008).

[15] S. Glied, Can. Math. Bull. 55, 98 (2011).

[16] P. Zeiner, Z. Kristallogr. 221, 105 (2006).

[17] P. Zeiner, Z. Kristallogr. 220, 915 (2005).

[18] P. Zeiner, in preparation. 